\title{
AN ADAPTIVE OBSERVER DESIGN APPROACH FOR A CLASS OF DISCRETE-TIME NONLINEAR SYSTEMS
}

\author{
KRISHNAN SRINIVASARENGAN ${ }^{a, *}$, JOSÉ RAGOT $^{a}$, CHRISTOPHE AUBRUN $^{a}$, \\ DIDIER MAQUIN ${ }^{a}$
}

\author{
${ }^{a}$ CRAN UMR 7039, CNRS \\ University of Lorraine, F-54000 Vandoeuvre-lès-Nancy, France \\ e-mail: \{krishnan.srinivasarengan, jose.ragot\}@univ-lorraine.fr \\ \{christophe.aubrun, didier.maquin\}@univ-lorraine.fr
}

\begin{abstract}
We consider the problem of joint estimation of states and some constant parameters for a class of nonlinear discrete-time systems. This class contains systems that could be transformed into a quasi-LPV (linear parameter varying) polytopic model in the Takagi-Sugeno (T-S) form. Such systems could have unmeasured premise variables, a case usually overlooked in the observer design literature. We assert that, for such systems in discrete-time, the current literature lacks design strategies for joint state and parameter estimation. To this end, we adapt the existing literature on continuous-time linear systems for joint state and time-varying parameter estimation. We first develop the discrete-time version of this result for linear systems. A Lyapunov approach is used to illustrate stability, and bounds for the estimation error are obtained via the bounded real lemma. We use this result to achieve our objective for a design procedure for a class of nonlinear systems with constant parameters. This results in less conservative conditions and a simplified design procedure. A basic waste water treatment plant simulation example is discussed to illustrate the design procedure.
\end{abstract}

Keywords: adaptive observer, joint state and parameter estimation, Takagi-Sugeno model, time-varying parameter estimation, sector nonlinearity transformation, discrete-time nonlinear systems.

\section{Introduction}

The term 'adaptive observers' is used to represent those observers that simultaneously estimate the states and unknown parameters (constant or time-varying) without augmenting the parameters along with the states. For nonlinear systems, a systematic procedure was first proposed by Cho and Rajamani (1997). However, the extension of this approach to discrete-time systems is not straightforward as it exploits some specific structure arising from continuous-time trajectories investigated in the proof.

Specific applications such as fault diagnosis have been attacked using adaptive observers as in the work of Caccavale et al. (2008), where a diagnostic observer adaptively estimates uncertainties. The authors assume all states as measured and use a peculiar innovation term $\mathbf{e}_{x, k+1}-\left(A-K_{o}\right) \mathbf{e}_{x, k}$, where $A$ and $K_{o}$ are the system and the state observer gain matrices, respectively, with

\footnotetext{
*Corresponding author
}

$\mathbf{e}_{x, k}$ representing the state error. This innovation allows cancellation of terms that complicates the adaptation of the approach by Cho and Rajamani (1997) to discrete-time. Another fault detection application based on the design of an adaptive observer is proposed by Thumati and Sarangapani (2008). The specific constraints used to tune the observers for the fault detection case do not extend to general adaptive observers.

One way to develop observers for general nonlinear systems could be using equivalent forms. A large class of nonlinear systems could be converted to linear parameter varying (LPV) or quasi-LPV system formulations. A nonlinear state equation of the form

$$
\mathbf{x}_{k+1}=f\left(\mathbf{x}_{k}, \mathbf{u}_{k}\right)
$$

could be put through a systematic procedure of factorizing them as proposed by Kwiatkowski et al. (2006) to obtain a quasi-LPV form,

$$
\mathbf{x}_{k+1}=A\left(\mathbf{x}_{k}, \mathbf{u}_{k}\right) \mathbf{x}_{k}+B\left(\mathbf{x}_{k}, \mathbf{u}_{k}\right) \mathbf{u}_{k} .
$$


For the model structure used by Cho and Rajamani (1997), the quasi-LPV form with unknown parameters $\theta_{j}, j=$ $1, \ldots, n_{\theta}$, would be

$$
\mathbf{x}_{k+1}=\sum_{j=1}^{n_{\theta}}\left(A_{j}\left(\mathbf{x}_{k}, \mathbf{u}_{k}\right) \mathbf{x}_{k} \theta_{j}+B_{j}\left(\mathbf{x}_{k}, \mathbf{u}_{k}\right) \mathbf{u}_{k} \theta_{j}\right),
$$

and possibly with an affine term. Here, $n_{\theta}$ is the number of parameters. If the system matrices depend only on measured or known variables, the observer design strategies for these systems are the same as those for linear time-varying (LTV) systems (Ţiclea and Besançon, 2016).

In that direction, we can also consider the adaptive observer proposed for LTV systems by Guyader and Zhang (2003). The authors set forth an innovation term whose gain is obtained by filtering the parameters' transmission matrix in the state equation. This structure, along with some boundedness assumption, allows showing the exponential convergence of the observer. If the system has bounded zero mean noise, the estimation errors have the expected value that exponentially converges to zero. The main issue with this approach is the lack of a clear procedure to choose a scalar that helps to guarantee convergence. These criticisms lead Ţiclea and Besançon (2016) to propose an exponential forgetting factor based approach. It mimics a Kalman filter with an update and propagation step, but has two interconnected exponential forgetting factor designs, thus preserving the adaptive observer structure. The main assumptions are complete uniform observability of the system and the invertibility of the system matrix of LTV, $A_{k}, \forall k$.

LTV based observers, however, cannot handle the case when the system matrices depend on one of the unmeasured states. This type of design can be handled in the realm of one of the quasi-LPV polytopic models: the Takagi-Sugeno (T-S) form. One way to obtain a $\mathrm{T}-\mathrm{S}$ model that exactly represents the original nonlinear system within a sector is using sector nonlinearity (SNL) transformation (Ohtake et al., 2003). For the quasi-LPV model in (3), applying SNL would lead to a T-S model of the form

$$
\begin{aligned}
\mathbf{x}_{k+1}= & \sum_{i=1}^{2^{n_{p}}} \mu_{i}\left(\mathbf{z}_{k}\right)\left[\left(A_{i}+\sum_{j=1}^{n_{\theta}} \bar{A}_{i j} \theta_{j}\right) \mathbf{x}_{k}\right. \\
& \left.\left.+\left(B_{i}+\sum_{j=1}^{n_{\theta}} \bar{B}_{i j} \theta_{j}\right)\right) \mathbf{u}_{k}\right] \\
\mathbf{y}_{k}= & C \mathbf{x}_{k},
\end{aligned}
$$

where $n_{p}$ is the number of premise variables $\mathbf{z}_{k}$, which could be one of the states, inputs, and outputs. Like Cho and Rajamani (1997), we consider here a linear output equation. We assert that observer design for such models can cover all possible systems that are represented by those in the work of Cho and Rajamani (1997). The observer for this type of system should take into account the fact that the weighting functions $\mu_{i}$ would be depending on estimated premise variables, rather than exact ones. Fortunately, there is a growing body of literature for observer design for T-S systems with unmeasured premise variables (Lendek et al., 2010), as well as using immersion techniques to avoid T-S systems with unmeasured premise variables (Ichalal et al., 2016).

The approach used in this paper springs from the idea of Bezzaoucha et al. (2013b) to represent a time-varying parameter using SNL transformation. This was extended to $\mathrm{T}-\mathrm{S}$ models with time-varying parameters by Bezzaoucha et al. (2013a). We derived the discrete-time version for T-S models (Srinivasarengan et al., 2016a). In the present paper, we first derive time-varying parameter estimation for a discrete-time linear system. Further, we assume the case of constant parameters and derive a less conservative and simpler design approach for nonlinear systems whose T-S form has unmeasured premise variables. In our opinion, this would fill the gap that is left in nonlinear discrete-time adaptive observers that cannot be solved by adapting LTV based design approaches.

This work builds on top of our previous communication (Srinivasarengan et al., 2016a). The key improvements show the generalized nature of the results for both LTV and T-S systems, providing extensions and refinement through corollaries, and proposing an adaptive observer design for constant parameters based on a simpler design approach with less conservative conditions. The illustration involves a different, but more relevant example. The outline of the paper is as follows: The following section discusses the preliminaries that are used later on. Section 3 discusses the model structure idea and formulates the problem. Joint state and time-varying parameters are derived for a discrete-time linear time-varying system in Section 4 These results are customized to design an adaptive observer for nonlinear discrete-time systems with constant parameters in Section [5. A simulation example is given in Section 6 to illustrate the proposed method. The paper is then summarized with a future outlook in Section 7 .

\section{Preliminaries}

2.1. Notation. Takagi-Sugeno models (Tanaka and Wang, 2004) are of the form

$$
\begin{aligned}
\mathbf{x}_{k+1} & =\sum_{i=1}^{r} \mu_{i}\left(\mathbf{z}_{k}\right)\left[A_{i} \mathbf{x}_{k}+B_{i} \mathbf{u}_{k}\right] \\
\mathbf{y}_{k} & =C \mathbf{x}_{k} .
\end{aligned}
$$


Here, $r=2^{n_{p}}$, where $n_{p}$ is the number of premise variables $\mathbf{z}_{k}$. The weighting functions $\mu_{i}\left(\mathbf{z}_{k}\right)$ capture the nonlinearity associated with the corresponding premise variables. Further,

$$
\mathbf{x}_{k} \in \mathbb{R}^{n_{x}}, \quad \mathbf{u}_{k} \in \mathbb{R}^{n_{u}}, \quad \mathbf{z}_{k} \in \mathbb{R}^{n_{p}}, \quad \mathbf{y}_{k} \in \mathbb{R}^{n_{y}}
$$

and

$$
A_{i} \in \mathbb{R}^{n_{x} \times n_{x}}, \quad B_{i} \in \mathbb{R}^{n_{x} \times n_{u}},
$$

$$
C \in \mathbb{R}^{n_{y} \times n_{x}}, \quad \forall i
$$

Given a symmetric matrix,

$$
A=\left[\begin{array}{cc}
a_{11} & a_{12} \\
* & a_{22}
\end{array}\right],
$$

the ' $*$ ' symbol represents the symmetric transpose element, that is, in this case, $*=a_{12}^{T}$.

2.2. Preliminary results. The following known results would be referred to while proving the results in this work.

Lemma 1. (Boyd et al., 1994) For a symmetric matrix $M$, given by

$$
M=\left[\begin{array}{cc}
A & B \\
B^{T} & C
\end{array}\right]
$$

if $C$ is invertible, then the following properties hold:

1. $M>0$ iff $C>0$ and $A-B C^{-1} B^{T}>0$;

2. if $C>0$, then $M \geq 0$ iff $A-B C^{-1} B^{T} \geq 0$.

Lemma 2. (Zhou and Khargonekar, 1988) Consider two matrices $X$ and $Y$ with appropriate dimensions, a timevarying matrix $\Delta_{k}$ and a positive scalar $\lambda$. Then

$$
X^{T} \Delta_{k}^{T} Y+Y^{T} \Delta_{k} X \leq \lambda X^{T} X+\lambda^{-1} Y^{T} Y
$$

for $\Delta_{k}^{T} \Delta_{k} \leq I$

Lemma 3. (de Souza and Xie, 1992) For a discrete-time system of the form

$$
\begin{aligned}
\mathbf{x}_{k+1} & =A \mathbf{x}_{k}+B \mathbf{u}_{k}, \\
\mathbf{y}_{k} & =C \mathbf{x}_{k}+D \mathbf{u}_{k},
\end{aligned}
$$

the bounded real lemma equivalent LMI condition for stability is

$$
\begin{gathered}
P=P^{T}>0 \\
{\left[\begin{array}{cc}
A^{T} P A-P+C^{T} C & A^{T} P B+C^{T} D \\
B^{T} P A+D^{T} C & D^{T} D+B^{T} P B-\Gamma_{2}
\end{array}\right] \leq 0}
\end{gathered}
$$

where $\Gamma_{2}$ is the $\mathbb{L}_{2}$ gain between the input $\mathbf{u}_{k}$ and the output $\mathbf{y}_{k}$.

\section{System model and problem formulation}

3.1. Representing a time-varying parameter using SNL. The idea of the estimation of a time-varying parameter lies in representing it using sector nonlinearity (SNL) transformation. SNL assumes that the parameter is bounded and its boundary values are known. For a scalar parameter $\theta_{k} \in\left[\theta^{1}, \theta^{2}\right]$, we could write

$$
\theta_{k}=\mu^{1}\left(\theta_{k}\right) \theta^{1}+\mu^{2}\left(\theta_{k}\right) \theta^{2},
$$

where

$$
\mu^{1}\left(\theta_{k}\right)=\frac{\theta^{2}-\theta_{k}}{\theta^{2}-\theta^{1}}, \quad \mu^{2}\left(\theta_{k}\right)=\frac{\theta_{k}-\theta^{1}}{\theta^{2}-\theta^{1}} .
$$

The membership functions satisfy the convex sum property, that is,

$$
\sum_{i} \mu^{i}(\cdot)=1, \quad 0 \leq \mu^{i}(\cdot) \leq 1, \quad \forall i
$$

Hence each parameter could be represented by a weighted sum of two elements.

Remark 1. For a vector case, or for $\mathrm{T}-\mathrm{S}$ systems with unknown parameters, the membership functions can be manipulated to obtain weighting functions that depend on the same membership functions. To illustrate this, take the case of two unknown parameters, $\theta_{1, k} \in\left[\theta_{1}^{1}, \theta_{1}^{2}\right]$ and $\theta_{2, k} \in\left[\theta_{2}^{1}, \theta_{2}^{2}\right]$, represented by

$$
\begin{aligned}
& \theta_{1, k}=\mu_{1}^{1}\left(\theta_{1, k}\right) \theta_{1}^{1}+\mu_{1}^{2}\left(\theta_{1, k}\right) \theta_{1}^{2}, \\
& \theta_{2, k}=\mu_{2}^{1}\left(\theta_{2, k}\right) \theta_{2}^{1}+\mu_{2}^{2}\left(\theta_{2, k}\right) \theta_{2}^{2} .
\end{aligned}
$$

We can now create a new formulation for the unknown parameters by

$$
\begin{aligned}
& \theta_{1, k}=\left(\mu_{2}^{1}\left(\theta_{2, k}\right)+\mu_{2}^{2}\left(\theta_{2, k}\right)\right) \theta_{1, k}, \\
& \theta_{2, k}=\left(\mu_{1}^{1}\left(\theta_{1, k}\right)+\mu_{1}^{2}\left(\theta_{1, k}\right)\right) \theta_{2, k}
\end{aligned}
$$

By bringing the alternative form in (12), we obtain the membership functions that depend on the same but four weighting functions, which are the products of the membership functions of the original representation. In general, this approach would lead to $2^{n_{\theta}}$ submodels, where $n_{\theta}$ is the number of parameters. A detailed treatment of this representation could be obtained from the work of Nagy et al. (2010).

3.2. System model structures. Consider the following linear time-varying system:

$$
\begin{aligned}
\mathbf{x}_{k+1} & =A\left(\Theta_{k}\right) \mathbf{x}_{k}+B\left(\Theta_{k}\right) \mathbf{u}_{k}, \\
\mathbf{y}_{k} & =C \mathbf{x}_{k},
\end{aligned}
$$

where $\Theta_{k} \in \mathbb{R}^{n_{\theta}}$ is the vector of the time-varying parameter $\theta_{i, k}, \forall i \in\left[1, \ldots, n_{\theta}\right], k$ being the time 
index. We consider only the following specific form of time-varying matrices:

$$
\begin{aligned}
& A\left(\Theta_{k}\right)=A_{0}+\sum_{i=1}^{n_{\theta}} \theta_{i, k} \bar{A}_{i}, \\
& B\left(\Theta_{k}\right)=B_{0}+\sum_{i=1}^{n_{\theta}} \theta_{i, k} \bar{B}_{i} ;
\end{aligned}
$$

that is, it is possible to write these time-varying matrix as a sum of constant matrices that are scaled by unknown parameters.

We can use SNL transformation as in (9) to represent the matrices of time-varying parameters. Let us first consider the scalar framework,

$$
\begin{aligned}
A\left(\theta_{k}\right) & =A_{0}+\theta_{k} \bar{A} \\
& =A_{0}+\left(\mu^{1}\left(\theta_{k}\right) \theta^{1}+\mu^{2}\left(\theta_{k}\right) \theta^{2}\right) \bar{A} \\
& =\sum_{j=1}^{2} \mu^{j}\left(\theta_{k}\right)\left(A_{0}+\theta^{j}\right) \bar{A},
\end{aligned}
$$

with $\theta^{j}$ corresponding to one of $\theta^{1}$ or $\theta^{2}$ depending upon the submodel $j$. Similarly,

$$
B\left(\theta_{k}\right)=\sum_{j=1}^{2} \mu^{j}\left(\theta_{k}\right)\left(B_{0}+\theta^{j}\right) \bar{B} .
$$

This could then be extended to the vector case to yield

$$
\begin{aligned}
A\left(\Theta_{k}\right) & =\sum_{i=1}^{2^{n_{\theta}}} \sum_{j=1}^{2} h_{i}\left(\Theta_{k}\right)\left(A_{0}+\theta_{i}^{j} \bar{A}_{i}\right), \\
B\left(\Theta_{k}\right) & =\sum_{i=1}^{2^{n_{\theta}}} \sum_{j=1}^{2} h_{i}\left(\Theta_{k}\right)\left(B_{0}+\theta_{i}^{j} \bar{B}_{i}\right),
\end{aligned}
$$

where $h_{i}\left(\Theta_{k}\right)$ is the normalized product of a membership function $\mu_{i}^{j}\left(\theta_{i, k}\right)$ of each parameter (see Remark 1) and $\theta_{i}^{j}, j=1,2$ represents the sector boundary values of each parameter $\theta_{i}$. Here $\theta_{j}^{i}$ is the corresponding maximum or minimum value of $\theta_{j}$ for the submodel $i$; more details could be obtained from Tanaka and Wang (2004). This would lead to

$$
\begin{aligned}
\mathbf{x}_{k+1} & =\sum_{i=1}^{r} h_{i}\left(\Theta_{k}\right)\left(A_{i} \mathbf{x}_{k}+B_{i} \mathbf{u}_{k}\right), \\
\mathbf{y}_{k} & =C \mathbf{x}_{k},
\end{aligned}
$$

with

$$
A_{i}=A_{0}+\sum_{j=1}^{n_{\theta}} \theta_{j}^{i} \bar{A}_{i}, \quad B_{i}=B_{0}+\sum_{j=1}^{n_{\theta}} \theta_{j}^{i} \bar{B}_{i} .
$$

For the model in (19), we propose an observer of the form

$$
\begin{aligned}
\hat{\mathbf{x}}_{k+1} & =\sum_{i=1}^{r} h_{i}\left(\hat{\Theta}_{k}\right)\left(A_{i} \hat{\mathbf{x}}_{k}+B_{i} \mathbf{u}_{k}+L_{i}\left(\mathbf{y}_{k}-\hat{\mathbf{y}}_{k}\right)\right), \\
\hat{\Theta}_{k+1} & =\hat{\Theta}_{k}+\sum_{i=1}^{r} h_{i}\left(\hat{\Theta}_{k}\right)\left(K_{y, i}\left(\mathbf{y}_{k}-\hat{\mathbf{y}}_{k}\right)-K_{\theta} \hat{\Theta}_{k}\right), \\
\hat{\mathbf{y}}_{k} & =C \hat{x}_{k} .
\end{aligned}
$$

The gains $L_{i} \in \mathbb{R}^{n_{x} \times n_{y}}$ and $K_{y, i} \in \mathbb{R}^{n_{\theta} \times n_{y}}$ are to be estimated while the gain $K_{\theta} \in \mathbb{R}^{n_{\theta} \times n_{\theta}}$ is chosen. The choice of $K_{\theta}$ will typically be in the form of a diagonal matrix. In the initial work (Bezzaoucha et al., 2013a), this was introduced to avoid a marginal stability condition for the error dynamics. As discussed by Srinivasarengan et al. (2016b), choosing this reduces the number of variables in the final LMI to be solved and hence allows a computationally tractable problem. Further, in the discrete-time case, $K_{\theta}$ as a variable leads to unresolvable nonlinear terms in the matrix inequalities.

3.3. Uncertain-like model representation. Let us define the state estimation error $\mathbf{e}_{\mathbf{x}, k}=\mathbf{x}_{k}-\hat{\mathbf{x}}_{k}$. If we want to analyse the dynamics of the errors based on the system and observer models in (19) and (21), it would involve comparing systems weighted by functions that depend on mismatched variables (i.e., $\mathbf{x}_{k}, \Theta_{k}$ vs $\hat{\mathbf{x}}_{k}, \hat{\Theta}_{k}$ ). This is a typical problem in observer design for T-S systems with unmeasured premise variables. There are various approaches to deal with it. In this work, we use the one proposed by Ichalal et al. (2009) to develop an uncertain-like model representation. Making use of the convex sum property in (11), we can rewrite (19), without making any approximations, as

$$
\begin{aligned}
\mathbf{x}_{k+1}= & \sum_{i=1}^{r} h_{i}\left(\hat{\Theta}_{k}\right)\left(A_{i} \mathbf{x}_{k}+B_{i} \mathbf{u}_{k}\right) \\
& +\sum_{i=1}^{r}\left(h_{i}\left(\Theta_{k}\right)-h_{i}\left(\hat{\Theta}_{k}\right)\right)\left(A_{i} \mathbf{x}_{k}+B_{i} \mathbf{u}_{k}\right), \\
\mathbf{y}_{k}= & C \mathbf{x}_{k} .
\end{aligned}
$$

Write

$$
\begin{aligned}
\Delta A_{k} & =\sum_{i=1}^{r}\left(h_{i}\left(\Theta_{k}\right)-h_{i}\left(\hat{\Theta}_{k}\right)\right) A_{i}=\mathcal{A} \Sigma_{A, k} E_{A}, \\
\Delta B_{k} & =\sum_{i=1}^{r}\left(h_{i}\left(\Theta_{k}\right)-h_{i}\left(\hat{\Theta}_{k}\right)\right) B_{i}=\mathcal{B} \Sigma_{B, k} E_{B},
\end{aligned}
$$

where

$$
\begin{aligned}
\mathcal{A} & =\left[\begin{array}{llll}
A_{1} & A_{2} & \ldots & A_{r}
\end{array}\right] \in \mathbb{R}^{n_{x} \times n_{x} r}, \\
E_{A} & =\left[\begin{array}{llll}
I_{n_{x}} & I_{n_{x}} & \cdots & I_{n_{x}}
\end{array}\right]^{T} \in \mathbb{R}^{n_{x} r \times n_{x}},
\end{aligned}
$$




$$
\Sigma_{A, k}=\left[\begin{array}{ccc}
\left(h_{1}-\hat{h}_{1}\right) I_{n_{x}} & \ldots & 0 \\
0 & & \left(h_{r}-\hat{h}_{r}\right) I_{n_{x}}
\end{array}\right] .
$$

Likewise,

$$
\begin{aligned}
& \mathcal{B}=\left[\begin{array}{llll}
B_{1} & B_{2} & \ldots & B_{r}
\end{array}\right] \in \mathbb{R}^{n_{u} \times n_{u} r}, \\
& E_{B}=\left[\begin{array}{llll}
I_{n_{u}} & I_{n_{u}} & \ldots & I_{n_{u}}
\end{array}\right]^{T} \in \mathbb{R}^{n_{u} r \times n_{u}}, \\
& \Sigma_{B, k}=\left[\begin{array}{ccc}
\left(h_{1}-\hat{h}_{1}\right) I_{n_{u}} & \ldots & 0 \\
0 & & \left(h_{r}-\hat{h}_{r}\right) I_{n_{u}}
\end{array}\right] \text {, }
\end{aligned}
$$

with $h_{i}$ and $\hat{h}_{i}$ standing for $h_{i}(\Theta)$ and $\hat{h}_{i}(\Theta)$, respectively. Since $-1 \leq\left(h_{i}-\hat{h}_{i}\right) \leq 1$, the matrices $\Sigma_{A, k} \in$ $\mathbb{R}^{n_{x} r \times n_{x} r}, \Sigma_{B, k} \in \mathbb{R}^{n_{u} r \times n_{u} r}$ have the useful property

$$
\Sigma_{A, k}^{T} \Sigma_{A, k} \leq I, \quad \Sigma_{B, k}^{T} \Sigma_{B, k} \leq I
$$

which will later be used to bound the time-varying difference between the known and estimated weighting functions. This will transform the system model (19) to the following representation:

$$
\begin{aligned}
\mathbf{x}_{k+1} & =\sum_{i=1}^{r} h_{i}(\hat{\Theta})\left[\left(A_{i}+\Delta A_{k}\right) \mathbf{x}_{k}+\left(B_{i}+\Delta B_{k}\right) \mathbf{u}_{k}\right], \\
\mathbf{y}_{k} & =C \mathbf{x}_{k} .
\end{aligned}
$$

As the model (27) and its observer (21) now share the same weighting functions $h_{i}(\hat{\Theta})$, it is therefore possible to express the state and the parameter estimation errors in a simpler and more tractable form.

\section{Joint state and time-varying parameter estimation}

In this section, we provide the results for stability analysis of the joint state and parameter observer. The results can be considered a discrete-time version of the observer design of Bezzaoucha et al. (2013a) and our approach follows the steps of Srinivasarengan et al. (2016a).

Theorem 1. Given the system model of the form (19), there exists an observer of the form (21) if there exist $P_{0}, P_{1}, R_{i}, F_{i}, \lambda_{1}, \lambda_{2}, \lambda_{3}, \lambda_{4}, \Gamma_{2}^{j}(\forall i \in[1, r], \forall j \in$ $\{x, u, \theta, \Delta \theta\})$ such that

$$
\begin{gathered}
P_{0}=P_{0}^{T}>0, \quad P_{1}=P_{1}^{T}>0, \\
\lambda_{m}>0, \forall m \in\{1,2,3,4\}, \quad \Gamma_{2}^{j}>0, \forall j, \\
{\left[\begin{array}{ccccc}
-P+I & Q_{A, i} & \Phi_{i}^{T} P & 0 & \\
* & T_{22} & 0 & Q_{B}^{T} & T_{\mathcal{A B}, i} \\
* & * & -P & 0 & \\
* & * & * & -P & \\
& * & & & \Lambda
\end{array}\right]<0 .}
\end{gathered}
$$

Here

$$
\begin{aligned}
& P_{0, i}=A_{i}^{T} P_{0}-C^{T} R_{i}^{T}, \quad P=\operatorname{diag}\left(P_{0}, P_{1}\right), \\
& \Phi_{i}^{T} P=\left[\begin{array}{cc}
P_{0, i} & -C^{T} F_{i}^{T} \\
0 & -K_{\theta}^{T} P_{1}
\end{array}\right], \\
& Q_{A, i}=\left[\begin{array}{cccc}
0 & 0 & -C^{T} F_{i}^{T}\left(I+K_{\theta}\right) & -C^{T} F_{i}^{T} \\
0 & 0 & -K_{\theta}^{T} P_{1}\left(I+K_{\theta}\right) & -K_{\theta}^{T} P_{1}
\end{array}\right] \text {, } \\
& Q_{B}=\left[\begin{array}{cc}
0 & 0 \\
0 & 0 \\
0 & \left(I+K_{\theta}\right)^{T} P_{1} \\
0 & P_{1}
\end{array}\right], \\
& T_{22}=\left[\begin{array}{cccc}
T_{22}^{11} & 0 & 0 & 0 \\
0 & T_{22}^{22} & 0 & 0 \\
0 & 0 & -\Gamma_{2}^{\theta} & 0 \\
0 & 0 & 0 & -\Gamma_{2}^{\Delta \theta}
\end{array}\right] \\
& \Lambda=\operatorname{diag}\left(-\lambda_{1} I,-\lambda_{3} I,-\lambda_{2} I,-\lambda_{4} I\right), \\
& T_{\mathcal{A B}, i}=\left[\begin{array}{cccc}
P_{0, i} \mathcal{A} & P_{0, i} \mathcal{B} & 0 & 0 \\
0 & 0 & 0 & 0 \\
0 & 0 & 0 & 0 \\
0 & 0 & P_{0} \mathcal{A} & P_{0} \mathcal{B}
\end{array}\right] \text {, }
\end{aligned}
$$

where $T_{22}^{11}=-\Gamma_{2}^{x}+\left(\lambda_{1}+\lambda_{2}\right) E_{A}^{T} E_{A}$ and $T_{22}^{22}=-\Gamma_{2}^{u}+$ $\left(\lambda_{3}+\lambda_{4}\right) E_{B}^{T} E_{B}$. The observer gains are given by

$$
K_{y, i}=P_{1}^{-1} F_{i}, \quad L_{i}=P_{0}^{-1} R_{i} .
$$

Proof. Consider the uncertain-like representation of the system in 27). Comparing it with the observer (21) and defining the errors

$$
\mathbf{e}_{\mathbf{x}, k}=\mathbf{x}_{k}-\hat{\mathbf{x}}_{k}, \quad \mathbf{e}_{\Theta, k}=\Theta_{k}-\hat{\Theta}_{k},
$$

we get

$$
\begin{aligned}
\mathbf{e}_{\mathbf{x}, k+1}= & \sum_{i=1}^{r} h_{i}\left(\hat{\Theta}_{k}\right)\left[\left(A_{i}-L_{i} C\right) \mathbf{e}_{\mathbf{x}, k}+\Delta A_{k} \mathbf{x}_{k}\right. \\
& \left.+\Delta B_{k} \mathbf{u}_{k}\right] \\
\mathbf{e}_{\Theta, k+1}= & \sum_{i=1}^{r} h_{i}\left(\hat{\Theta}_{k}\right)\left[\Delta \Theta_{k}+\left(I+K_{\theta}\right) \theta_{k}\right. \\
& \left.-K_{y, i} C \mathbf{e}_{\mathbf{x}, k}-K_{\theta} \mathbf{e}_{\Theta, k}\right],
\end{aligned}
$$

where $\Delta \Theta_{k}=\Theta_{k+1}-\Theta_{k}$. We can represent these error dynamics as

$$
\begin{aligned}
& {\left[\begin{array}{c}
\mathbf{e}_{\mathbf{x}, k+1} \\
\mathbf{e}_{\Theta, k+1}
\end{array}\right]} \\
& \quad=\sum_{i=1}^{r} h_{i}\left(\hat{\Theta}_{k}\right)\left(\Phi_{i}\left[\begin{array}{c}
\mathbf{e}_{\mathbf{x}, k} \\
\mathbf{e}_{\Theta, k}
\end{array}\right]+\Psi_{i, k}\left[\begin{array}{c}
\mathbf{x}_{k} \\
\mathbf{u}_{k} \\
\Theta_{k} \\
\Delta \Theta_{k}
\end{array}\right]\right),
\end{aligned}
$$


where

$$
\begin{aligned}
\Phi_{i} & =\left[\begin{array}{cc}
A_{i}-L_{i} C & 0 \\
-K_{y, i} C & -K_{\theta}
\end{array}\right] \\
\Psi_{i, k} & =\left[\begin{array}{cccc}
\Delta A_{k} & \Delta B_{k} & 0 & 0 \\
0 & 0 & I+K_{\theta} & I
\end{array}\right] .
\end{aligned}
$$

Setting

$$
\begin{aligned}
& \mathbf{e}_{a, k}=\left[\begin{array}{ll}
\mathbf{e}_{\mathbf{x}, k}^{T} & \mathbf{e}_{\Theta, k}^{T}
\end{array}\right]^{T}, \\
& \tilde{u}_{k}=\left[\begin{array}{llll}
\mathbf{x}_{k}^{T} & \mathbf{u}_{k}^{T} & \Theta_{k}^{T} & \Delta \Theta_{k}^{T}
\end{array}\right]^{T},
\end{aligned}
$$

the error dynamics can be written as

$$
\mathbf{e}_{a, k+1}=\sum_{i=1}^{r} h_{i}\left(\hat{\Theta}_{k}\right)\left(\Phi_{i} \mathbf{e}_{a, k}+\Psi_{i, k} \tilde{u}_{k}\right) .
$$

The aim here is an asymptotic decay of the error and the minimization of the effect of $\tilde{u}_{k}$ on the error. It is to be noted that $\Phi_{i}$ has constant entries, but $\Psi_{i, k}$ has time-varying entries. To analyze the stability of (34), we consider the following Lyapunov candidate:

$$
V_{k}=\mathbf{e}_{a, k}^{T} P \mathbf{e}_{a, k} .
$$

Since there are time-varying perturbations that affect the error $\mathbf{e}_{a, k}$ in (34), the sufficient condition for stability that we consider is

$$
V_{k+1}<V_{k}-\left(\mathbf{e}_{a, k}^{T} \mathbf{e}_{a, k}-\tilde{u}_{k}^{T} \Gamma_{2} \tilde{u}_{k}\right),
$$

where $\Gamma_{2}$ is a block diagonal matrix with the entries

$$
\Gamma_{2}=\operatorname{diag}\left(\Gamma_{2}^{x}, \Gamma_{2}^{u}, \Gamma_{2}^{\theta}, \Gamma_{2}^{\Delta \theta}\right)
$$

that represent the $\mathbb{L}_{2}$-gains of the effect of the elements in $\tilde{u}$ on the error. Applying the discrete-time version of the bounded real lemma (BRL) in Lemma 3, we get the matrix inequality condition

$$
\left[\begin{array}{cc}
\Phi_{i}^{T} P \Phi_{j}-P+I & \Phi_{i}^{T} P \Psi_{i, k} \\
* & \Psi_{i, k}^{T} P \Psi_{l, k}-\Gamma_{2}
\end{array}\right]<0 .
$$

The introduction of $l$ terms is to illustrate that we have cross terms between the different submodels. However, we could take the more conservative condition of considering $l=i$, based on the illustrations in Theorem 17 by Blanco (2001). We find another form for (38) so as to

- obtain linear bounds for the nonlinearities (in $\Phi_{i}^{T} P \Phi_{j}, \Phi_{i}^{T} P \Psi_{i, k}$ and their transposes);

- obtain bounds for the time-varying terms (in $\Phi_{i}^{T} P \Psi_{i, k}$ and $\left.\Psi_{i, k}^{T} P \Psi_{j, k}\right)$.
Reducing nonlinearities. The quadratic terms associated with $\Phi_{i}$ and $\Psi_{i, k}$ could be reduced to linear terms. By using the Schur complements (Lemma 1) for the nonlinear terms, the matrix terms in (38) could be reduced to

$$
\left[\begin{array}{cccc}
-P+I & \Phi_{i}^{T} P \Psi_{i, k} & \Phi_{i}^{T} P & 0 \\
* & -\Gamma_{2} & 0 & \Psi_{i, k}^{T} P \\
* & * & -P & 0 \\
* & * & * & -P
\end{array}\right]<0 .
$$

This has not resolved all the nonlinear entries, though, and the residual factors are in the form of unresolvable terms inside $\Phi_{i}^{T} P$ and $\Phi_{i}^{T} P \Psi_{i, k}$. This is because, as in (33), $\Phi_{i}$ has two variables $L_{i}$ and $K_{y, i}$, as part of the matrix split into $n_{x}$ and $n_{\theta}$ blocks. This issue is alleviated in two steps:

- Consider a diagonal structure for the Lyapunov matrix $P=\left[\begin{array}{cc}P_{0} & 0 \\ 0 & P_{1}\end{array}\right]$.

- This Lyapunov structure would lead to terms $P_{0} L_{i}$ and $P_{1} K_{y, i}$. These quadratic terms are eliminated by introducing new variables,

$$
R_{i}=P_{0} L_{i}, \quad F_{i}=P_{1} K_{y, i}
$$

These steps would reduce the nonlinear matrix entries in (39) to linear terms. First, we define, to simplify the notation,

$$
P_{0, i}=A_{i}^{T} P_{0}-C^{T} R_{i}^{T} .
$$

The term $\Phi_{i}^{T} P$ would reduce to

$$
\Phi_{i}^{T} P=\left[\begin{array}{cc}
P_{0, i} & -C^{T} F_{i}^{T} \\
0 & -K_{\theta}^{T} P_{1}
\end{array}\right] .
$$

Further, we split the linearized time-varying matrices to those with constant entries and time-varying terms,

$$
\begin{gathered}
\Phi_{i}^{T} P \Psi_{i, k}=Q_{A, i}+\mathcal{L}_{U, i, k}, \\
\Psi_{i, k}^{T} P=Q_{B}+\mathcal{L}_{L, k}^{T},
\end{gathered}
$$

where

$$
\begin{aligned}
& Q_{A, i}=\left[\begin{array}{cccc}
0 & 0 & -C^{T} F_{i}^{T}\left(I+K_{\theta}\right) & -C^{T} F_{i}^{T} \\
0 & 0 & -K_{\theta}^{T} P_{1}\left(I+K_{\theta}\right) & -K_{\theta}^{T} P_{1}
\end{array}\right], \\
& Q_{B}=\left[\begin{array}{cc}
0 & 0 \\
0 & 0 \\
0 & \left(I+K_{\theta}\right)^{T} P_{1} \\
0 & P_{1}
\end{array}\right] \text {, } \\
& \mathcal{L}_{U, i, k}=\left[\begin{array}{cccc}
P_{0, i} \Delta A_{k} & P_{0, i} \Delta B_{k} & 0 & 0 \\
0 & 0 & 0 & 0
\end{array}\right] \text {, } \\
& \mathcal{L}_{L, k}=\left[\begin{array}{cccc}
P_{0} \Delta A_{k} & P_{0} \Delta B_{k} & 0 & 0 \\
0 & 0 & 0 & 0
\end{array}\right] \text {. }
\end{aligned}
$$


Bounds for time-varying terms. The linearized version of the inequality in (39) can now be split into terms with and without time-varying terms and their corresponding transposes,

$$
Q_{i}+\mathcal{L}_{i, k}+\mathcal{L}_{i, k}^{T}<0
$$

where

$$
Q_{i}=\left[\begin{array}{cccc}
-P+I & Q_{A, i} & \Phi_{i}^{T} P & 0 \\
* & -\Gamma_{2} & 0 & Q_{B} \\
* & * & -P & 0 \\
* & * & * & -P
\end{array}\right]
$$

with $Q_{A, i}$ and $Q_{B}$ given in (30). The time-varying terms are gathered as follows:

$$
\mathcal{L}_{i, k}=\left[\begin{array}{cccc}
0 & \mathcal{L}_{U, i, k} & 0 & 0 \\
0 & 0 & 0 & 0 \\
0 & 0 & 0 & 0 \\
0 & \mathcal{L}_{L, k} & 0 & 0
\end{array}\right]
$$

There are four time-varying terms and their transposes in (44). In (23), we showed that the uncertain-like terms could be written as products of matrices and further showed an interesting property of the time-varying matrix in (26). Bythe some token, we can split each of the uncertain-like terms in $\mathcal{L}_{i, k}$. Let us insert the four terms corresponding to the time-varying factors, $P_{0, i} \Delta A_{k}, P_{0} \Delta A_{k}, P_{0, i} \Delta B_{k}, P_{0} \Delta B_{k}$, as parts of individual matrices, $\mathcal{L}_{A 1, k}, \mathcal{L}_{A 2, k}, \mathcal{L}_{B 1, k}, \mathcal{L}_{B 2, k}$ respectively, that is,

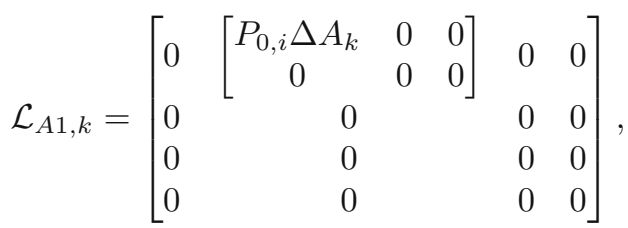

$$
\begin{aligned}
& \mathcal{L}_{B 2, k}=\left[\begin{array}{ccccc}
0 & & 0 & 0 & 0 \\
0 & 0 & 0 & 0 \\
0 & 0 & 0 & 0 \\
0 & {\left[\begin{array}{ccc}
0 & P_{0} \Delta B_{k} & 0 \\
0 & 0 & 0
\end{array}\right]} & 0 & 0
\end{array}\right],
\end{aligned}
$$

and similarly for $\mathcal{L}_{B 1, k}$ and $\mathcal{L}_{A 2, k}$, so that

$$
\mathcal{L}_{i, k}=\mathcal{L}_{A 1, k}+\mathcal{L}_{A 2, k}+\mathcal{L}_{B 1, k}+\mathcal{L}_{B 2, k} .
$$

It is to be noted that the zero entries in the matrices have appropriate dimensions and are usually grouped together to make the representation easier. Based on the representation in (23), we represent the uncertain-like terms as

$$
\begin{aligned}
& \left.\mathcal{L}_{A 1, k}=\left[\begin{array}{c}
P_{0, i} \mathcal{A} \\
0 \\
0 \\
0 \\
0
\end{array}\right] \Sigma_{A, k}\left[\begin{array}{llllll}
0 & {\left[E_{A}\right.} & 0 & 0
\end{array}\right] \quad 0 \quad 0\right] \\
& \left.\mathcal{L}_{A 2, k}=\left[\begin{array}{c}
0 \\
0 \\
0 \\
{\left[\begin{array}{c}
P_{0} \mathcal{A} \\
0
\end{array}\right]}
\end{array}\right] \Sigma_{A, k}\left[\begin{array}{llllll}
0 & {\left[E_{A}\right.} & 0 & 0
\end{array}\right] \quad 0 \quad 0\right], \\
& \mathcal{L}_{B 1, k}=\left[\begin{array}{c}
P_{0, i} \mathcal{B} \\
0 \\
0 \\
0 \\
0
\end{array}\right] \Sigma_{B, k}\left[\begin{array}{llllll}
0 & {\left[\begin{array}{lll}
0 & E_{B} & 0
\end{array}\right]} & 0 & 0
\end{array}\right], \\
& \mathcal{L}_{B 2, k}=\left[\begin{array}{c}
0 \\
0 \\
0 \\
{\left[\begin{array}{c}
P_{0} \mathcal{B} \\
0
\end{array}\right]}
\end{array}\right] \Sigma_{B, k}\left[\begin{array}{llllll}
0 & {\left[\begin{array}{lll}
0 & E_{B} & 0
\end{array}\right]} & 0 & 0
\end{array}\right] \text {. }
\end{aligned}
$$

Now, we apply Lemma 2 to the sum of these terms and their transposes,

$$
\begin{aligned}
& \mathcal{L}_{A 1, k}+\mathcal{L}_{A 1, k}^{T} \\
& \leq \lambda_{1}^{-1}\left[\begin{array}{c}
P_{0, i} \mathcal{A} \\
0 \\
0 \\
0 \\
0
\end{array}\right]\left[\left[\mathcal{A}^{T} P_{0, i} \quad 0\right] \quad 0 \quad 000\right] \\
& +\lambda_{1}\left[\begin{array}{c}
0 \\
E_{A}^{T} \\
0 \\
0 \\
0 \\
0
\end{array}\right]\left[\begin{array}{llllll}
0 & {\left[\begin{array}{llll}
E_{A} & 0 & 0
\end{array}\right]} & 0 & 0
\end{array}\right]
\end{aligned}
$$

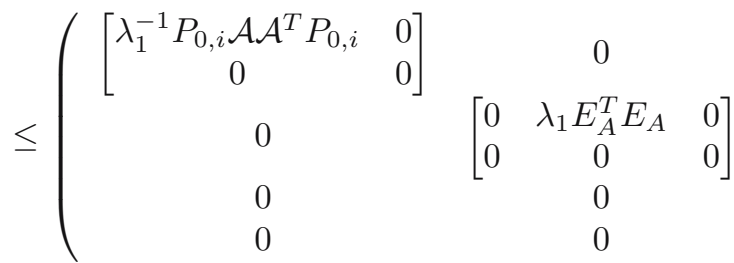

$$
\begin{aligned}
& \left.\begin{array}{ll}
0 & 0 \\
0 & 0 \\
0 & 0 \\
0 & 0
\end{array}\right)
\end{aligned}
$$

for some scalar $\lambda_{1}$. Similarly, the sums of $\mathcal{L}_{A 2, k}+$ $\mathcal{L}_{A 2, k}^{T}, \mathcal{L}_{B 1, k}+\mathcal{L}_{B 1, k}^{T}$ and $\mathcal{L}_{B 2, k}+\mathcal{L}_{B 2, k}^{T}$ are bounded 
(respectively) by

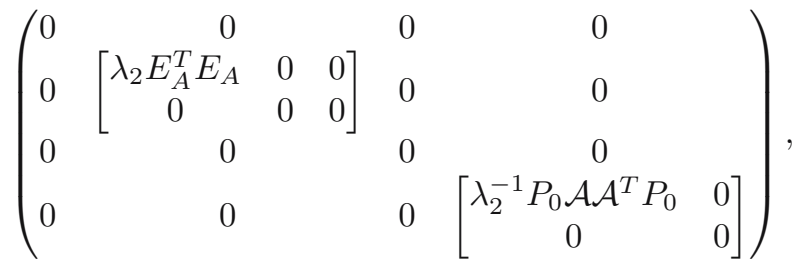

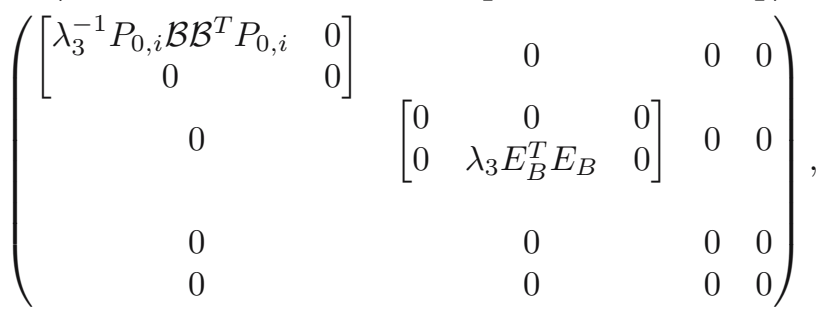

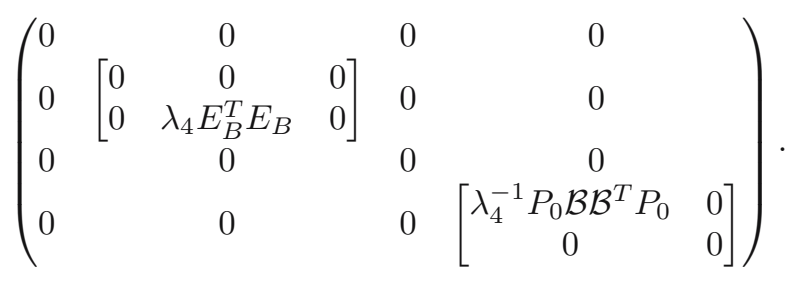

Adding them all up gives

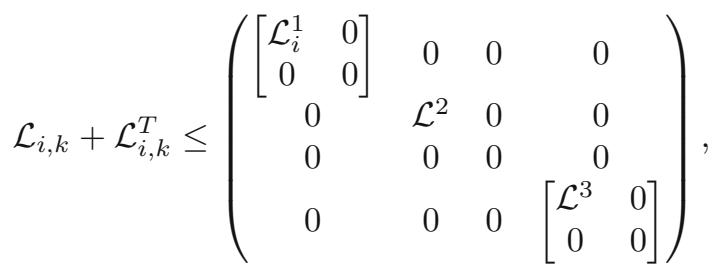

with

$$
\begin{aligned}
\mathcal{L}_{i}^{1} & =\lambda_{1}^{-1} P_{0, i} \mathcal{A} \mathcal{A}^{T} P_{0, i}+\lambda_{3}^{-1} P_{0, i} \mathcal{B B}^{T} P_{0, i}, \\
\mathcal{L}^{2} & =\left[\begin{array}{cccc}
\left(\lambda_{1}+\lambda_{2}\right) E_{A}^{T} E_{A} & 0 & 0 & 0 \\
0 & \left(\lambda_{3}+\lambda_{4}\right) E_{B}^{T} E_{B} & 0 & 0 \\
0 & 0 & 0 & 0 \\
0 & 0 & 0 & 0
\end{array}\right], \\
\mathcal{L}^{3} & =\lambda_{2}^{-1} P_{0} \mathcal{A} \mathcal{A}^{T} P_{0}+\lambda_{4}^{-1} P_{0} \mathcal{B B}^{T} P_{0} .
\end{aligned}
$$

This would lead the inequality in (44) to

$$
\left(\begin{array}{cccc}
\mathcal{L}_{i}^{11} & Q_{A, i} & \Phi_{i}^{T} P & 0 \\
* & -\Gamma_{2}+\mathcal{L}^{2} & 0 & Q_{B} \\
* & * & -P & 0 \\
* & * & * & \mathcal{L}^{44}
\end{array}\right)<0
$$

where

$$
\begin{aligned}
\mathcal{L}_{i}^{11} & =\left[\begin{array}{cc}
-P_{0}+I+\mathcal{L}_{i}^{1} & 0 \\
0 & P_{1}
\end{array}\right], \\
\mathcal{L}^{44} & =\left[\begin{array}{cc}
-P_{0}+I+\mathcal{L}^{3} & 0 \\
0 & P_{1}
\end{array}\right] .
\end{aligned}
$$

These terms have quadratic entries that could be handled by applying Schur's complement. In this way, we could consider

$$
\mathcal{L}_{i}^{11}<0 \Leftrightarrow\left[\begin{array}{cccc}
-P_{0}+I & 0 & P_{0, i} \mathcal{A} & P_{0, i} \mathcal{B} \\
0 & P_{1} & 0 & 0 \\
0 & 0 & -\lambda_{1} I & 0 \\
0 & 0 & 0 & -\lambda_{3} I
\end{array}\right]<0
$$

and in much the same way as we could do for $\mathcal{L}^{44}$. Putting them together and rearranging, we get

$$
\left[\begin{array}{ccccc}
-P+I & Q_{A, i} & \Phi_{i}^{T} P & 0 & \\
* & T_{22} & 0 & Q_{B}^{T} & T_{\mathcal{A B}, i} \\
* & * & -P & 0 & \\
* & * & * & -P & \\
& * & & & \Lambda
\end{array}\right]<0
$$

with

$$
\begin{aligned}
T_{\mathcal{A B}, i} & =\left[\begin{array}{cccc}
P_{0, i} \mathcal{A} & P_{0, i} \mathcal{B} & 0 & 0 \\
0 & 0 & 0 & 0 \\
0 & 0 & 0 & 0 \\
0 & 0 & P_{0} \mathcal{A} & P_{0} \mathcal{B}
\end{array}\right], \\
\Lambda & =\left[\begin{array}{cccc}
-\lambda_{1} I & 0 & 0 & 0 \\
0 & -\lambda_{3} I & 0 & 0 \\
0 & 0 & -\lambda_{2} I & 0 \\
0 & 0 & 0 & -\lambda_{4} I
\end{array}\right] .
\end{aligned}
$$

This completes the proof.

Corollary 1. We could formulate observer design as an optimization problem with the objective to minimize the $\mathbb{L}_{2}$-gain between the perturbation factors $\tilde{u}_{k}$ and the errors $\mathbf{e}_{a, k}$ in (34). We could aim to minimize a scalar $\beta$ such that

$$
\min _{P_{0}, P_{1}, F_{i}, R_{i}, \Gamma_{2}^{j}, \lambda_{m}} \beta
$$

$\forall i \in[1, r], \forall j \in\{x, u, \theta, \Delta \theta\}, \forall m \in\{1,2,3,4\}$, so that the LMIs in 29] are satisfied along with

$$
\beta I>\Gamma_{2}^{j}, \quad \forall j \in\{x, u, \theta, \Delta \theta\} .
$$

There is an inherent assumption that the $\mathbb{L}_{2}$-gains of various perturbations are scaled appropriately so that using a single $\beta$ makes sense. This could otherwise be achieved by using an appropriate scaling factor instead of I on the left hand side of (57).

Corollary 2. Measurement noise could be added to the output equation in (19) so that

$$
\mathbf{y}_{k}=C \mathbf{x}_{k}+H \nu_{k}
$$

where $\nu_{k} \in \mathbb{R}^{n_{y}}$ is the measurement noise with $H \in \mathbb{R}^{n_{y} \times n_{y}}$ the transmission matrix. This would 
make the perturbation variable turn into $\tilde{u}_{k}=$ $\left[\begin{array}{lllll}\mathbf{x}_{k} & \mathbf{u}_{k} & \Theta_{k} & \Delta \Theta_{k} & \nu_{k}\end{array}\right]^{T}$ and the matrix $\Psi_{i, k}$ in (33) into

$$
\Psi_{i, k}=\left[\begin{array}{ccccc}
\Delta A_{k} & \Delta B_{k} & 0 & 0 & -L_{i} H \\
0 & 0 & I+K_{\theta} & I & -K_{y, i} H
\end{array}\right],
$$

which would then lead to the same LMIs with the modifications in the following components in (30):

$$
\begin{gathered}
Q_{A, i} \\
{\left[\begin{array}{llllll}
0 & 0 & -C^{T} F_{i}^{T}\left(I+K_{\theta}\right) & -C^{T} F_{i}^{T} & -R_{i} H \\
0 & 0 & -K_{\theta}^{T} P_{1}\left(I+K_{\theta}\right) & -K_{\theta}^{T} P_{1} & -F_{i} H
\end{array}\right],} \\
T_{22}=\left[\begin{array}{ccccc}
T_{22}^{11} & 0 & 0 & 0 & 0 \\
0 & T_{22}^{22} & 0 & 0 & 0 \\
0 & 0 & -\Gamma_{2}^{\theta} & 0 & 0 \\
0 & 0 & 0 & -\Gamma_{2}^{\Delta \theta} & 0 \\
0 & 0 & 0 & 0 & -\Gamma_{2}^{\nu}
\end{array}\right],
\end{gathered}
$$

where $\Gamma_{2}^{\nu}$ is the $\mathbb{L}_{2}$-gain between the noise $\nu$ and the error $\mathbf{e}_{a, k}$. It is to be noted that $\Gamma_{2}^{\nu}$ will also be added as a diagonal block in the matrix $\Gamma_{2}$.

Remark 2. It is to be noted here that since there are only two time-varying terms $\Delta A_{k}$ and $\Delta B_{k}$ in (46), we could split the time-varying terms into only two additive factors, and hence apply Lemma 2 twice. However, the resulting matrix inequality is nonlinear with crossover terms, making it impossible to resolve. Hence four additive factors were used.

Remark 3. In the continuous-time version discussed by Bezzaoucha et al. (2013a), the factor $K_{\theta}$ allowed avoiding numerical issues in the LMI conditions. In our work, this has been followed through. The value of $K_{\theta}$, however, is also important because it may make the effect of the innovation term $K_{y, i}\left(\mathbf{y}_{k}-\hat{\mathbf{y}}_{k}\right)$ negligible due to relative scaling between $K_{\theta}$ and $K_{y, i}$, as discussed by Srinivasarengan et al. (2016b). This could be done by adding an extra condition. For example, for a scalar parameter estimation case, let $k_{\theta}$ be the scalar value of the observer gain $K_{\theta}$, which would yield the condition

$$
\frac{1}{k_{\theta}} K_{y, i}>\rho,
$$

where $\rho>1$ is a constant chosen depending upon the relative scaling between $\theta$ and $\mathbf{y}_{k}-\hat{\mathbf{y}}_{k}$. Along with the LMIs in 29], we could add, for a scalar case,

$$
F_{i}>\rho P_{1} k_{\theta} .
$$

Remark 4. The LMI in (29) could be considered restrictive partly because of the term $-P+I$ that calls for $P$ to be more positive than $I$. This starts with the term $e_{a, k}^{T} I e_{a, k}$ in the Lyapunov function trajectory in 36 . If a solution is unavailable for this case, we could replace this with $e_{a, k}^{T} Q_{e} e_{a, k}$, where $Q_{e}$ could be chosen to be a value that allows a solution to the LMI (29) to exist.

Remark 5. The extension of Theorem 1 to a nonlinear system represented by $\mathrm{T}-\mathrm{S}$ models is straightforward. That is, the nonlinear model has to be transformed into a $\mathrm{T}-\mathrm{S}$ model with time-varying matrices and then all the proposed development with the introduction of a supplementary index for some of the matrices involved. Furthermore, the weighting functions now would be the products of membership functions of both unknown parameters as well as the premise variables of the T-S model. Hence the components of the uncertain-like terms would be different.

\section{Adaptive observer design}

Consider now a discrete-time version of the nonlinear system by Cho and Rajamani (1997) and the particular case where the unknown parameter vector $\Theta$ is constant:

$$
\begin{aligned}
\mathbf{x}_{k+1} & =A \mathbf{x}_{k}+\phi\left(\mathbf{x}_{k}, \mathbf{u}_{k}\right)+b f\left(\mathbf{x}_{k}, \mathbf{u}_{k}\right) \Theta \\
\mathbf{y}_{k} & =C \mathbf{x}_{k}
\end{aligned}
$$

where a quasi-LPV equivalent would be of the form (4). Our aim is to design an adaptive observer for this model, assuming that we know a range of values $\left[\theta_{i}^{1}, \theta_{i}^{2}\right]$ in which the true value of each $\theta_{i}, \forall i \in 1, \ldots, n_{\theta}$ lies. This substitutes the sector bounds for the time-varying case. In much the same way as in the previous section, applying SNL transformation to the time-varying parameter with these bounds, we obtain

$$
\begin{aligned}
\mathbf{x}_{k+1} & =\sum_{i=1}^{s} h_{i}\left(\mathbf{z}_{k}, \Theta\right)\left(A_{i} \mathbf{x}_{k}+B_{i} \mathbf{u}_{k}\right) \\
\mathbf{y}_{k} & =C \mathbf{x}_{k}
\end{aligned}
$$

where $s=2^{n_{p}+n_{\theta}}$ and $h_{i}\left(\mathbf{z}_{k}, \Theta\right)$ is the weighting function obtained by normalizing the product of membership functions associated with the premise variables $\mathbf{z}_{k}$ and the parameters $\theta$. For this type of system, we propose an observer of the form

$$
\begin{aligned}
\hat{\mathbf{x}}_{k+1} & =\sum_{i=1}^{s} h_{i}\left(\hat{z}_{k}, \hat{\Theta}_{k}\right)\left[A_{i} \hat{x}+B_{i} \mathbf{u}_{k}+L_{i}\left(\mathbf{y}_{k}-\hat{\mathbf{y}}_{k}\right)\right] \\
\hat{\Theta}_{k+1} & =\hat{\Theta}_{k}+\sum_{i=1}^{s} h_{i}\left(\hat{z}_{k}, \hat{\Theta}_{k}\right) K_{y, i}\left(\mathbf{y}_{k}-\hat{\mathbf{y}}_{k}\right) \\
\hat{\mathbf{y}}_{k} & =C \mathbf{x}_{k} .
\end{aligned}
$$

As could be noted, the gain term $K_{\theta}$ has been dropped. One main reason is the simplification this offers (this will be apparent soon). Further, the condition $K_{\theta}=$ 
0 leads to unsolvable LMIs in the continuous-time case, but not in the discrete-time one. To compute the state and parameter error, we follow the uncertain-like model approach, with the augmented error dynamics given by

$$
\begin{aligned}
\mathbf{e}_{a, k+1}= & {\left[\begin{array}{cc}
A_{i}-L_{i} C & 0 \\
-K_{y, i} C & 0
\end{array}\right] \mathbf{e}_{a, k} } \\
& +\left[\begin{array}{cc}
\Delta A_{k} & \Delta B_{k} \\
0 & 0
\end{array}\right]\left[\begin{array}{l}
\mathbf{x}_{k} \\
\mathbf{u}_{k}
\end{array}\right]
\end{aligned}
$$

As could be seen, the number of perturbations has reduced and hence the dynamics matrices are simplified. By applying the discrete-time BRL and following it up with the application of LMI equivalence using Schur's complement (Lemma 1), then splitting the Lyapunov matrix to the form

$$
P=\left[\begin{array}{cc}
P_{0} & 0 \\
0 & P_{1}
\end{array}\right],
$$

and applying the variable transformations $R_{i}=P_{0} L_{i}$ and $F_{i}=P_{1} K_{y, i}$, we get

$$
\left[\begin{array}{cccc}
-P+I & \Phi_{i}^{T} P \Psi_{i, k} & \Phi_{i}^{T} P & 0 \\
* & -\Gamma_{2} & 0 & \Psi_{i, k}^{T} P \\
* & * & -P & 0 \\
* & * & * & -P
\end{array}\right]<0
$$

with

$$
\begin{aligned}
\Phi_{i}^{T} P \Psi_{i, k} & =\left[\begin{array}{cc}
P_{0, i} \Delta A_{k} & P_{0, i} \Delta B_{k} \\
0 & 0
\end{array}\right], \\
\Phi_{i}^{T} P & =\left[\begin{array}{cc}
P_{0, i} & -C^{T} F_{i}^{T} \\
0 & 0
\end{array}\right], \\
\Psi_{i, k}^{T} P & =\left[\begin{array}{cc}
\Delta A_{k}^{T} P_{0} & \Delta B_{k}^{T} P_{0} \\
0 & 0
\end{array}\right] .
\end{aligned}
$$

Furthermore, following the steps described in the proof of Theorem 1, we can summarize the results as follows.

Theorem 2. Given a nonlinear discrete-time system of the form 63. which can be transformed into a T-S model, an observer of the form (65) could be designed if there exist $P_{0}, P_{1}, R_{i}, F_{i}, \lambda_{1}, \lambda_{2}, \lambda_{3}, \lambda_{4}, \Gamma_{2}^{j}(\forall i \in[1, r], \forall j \in$ $\{x, u\})$ such that

$$
\begin{gathered}
P_{0}=P_{0}^{T}>0, \quad P_{1}=P_{1}^{T}>0, \\
\lambda_{m}>0, \forall m \in 1,2,3,4, \quad \Gamma_{2}^{j}>0, \forall j, \\
{\left[\begin{array}{cccc|c}
-P+I & 0 & \Phi_{i}^{T} P & 0 & \\
* & T_{22} & 0 & 0 & T_{\mathcal{A B}} \\
* & * & -P & 0 & \\
* & * & * & -P & \\
\hline * & & & \Lambda
\end{array}\right]<0,}
\end{gathered}
$$

where $\Phi_{i}^{T} P$ is given in (68) and $\Lambda$ in (30), $T_{\mathcal{A B}, i}$ has the same structure as in 30xcept for accommodating the
Table 1. Model parameters.

\begin{tabular}{|c|c|}
\hline Parameter & Value \\
\hline \hline$a_{0}$ & 0.5 \\
$b$ & 0.4 \\
$c$ & 0.4 \\
$d$ & 2 \\
$T_{s}$ & 1 \\
\hline
\end{tabular}

changes in the number of zero rows due to the change of $T_{22}$ to

$$
T_{22}=\left[\begin{array}{cc}
T_{22}^{11} & 0 \\
0 & T_{22}^{22}
\end{array}\right],
$$

where $T_{22}^{11}=-\Gamma_{2}^{x}+\left(\lambda_{1}+\lambda_{2}\right) E_{A}^{T} E_{A}$ and $T_{22}^{11}=-\Gamma_{2}^{u}+$ $\left(\lambda_{3}+\lambda_{4}\right) E_{B}^{T} E_{B}$. The observer gains are given by $K_{y, i}=$ $P_{1}^{-1} F_{i}$ and $L_{i}=P_{0}^{-1} R_{i}$.

Proof. The proof follows that of Theorem 1 with the changes in the matrix block entries discussed above.

\section{Simulation example}

We consider a discrete-time version of the simplified waste water treatment plant from the work of Bezzaoucha et al. (2013a). The simplification concerns reducing the 10 -state system to a 2-state model, given by

$$
\begin{aligned}
x_{1, k+1} & =x_{1, k}+T_{s}\left[\frac{a x_{1, k}}{x_{2, k}+b} x_{2, k}-x_{1, k} u_{k}\right], \\
x_{2, k+1} & =x_{2, k}+T_{s}\left[-\frac{c a x_{1, k}}{x_{2, k}+b} x_{2, k}+\left(d-x_{2, k}\right) u_{k}\right], \\
y_{k} & =x_{1, k} .
\end{aligned}
$$

In this model, we consider an uncertainty in the parameter $a$, that is,

$$
a=a_{0}+\theta \text {. }
$$

The parameters of the model used are given in Table 1 The unknown parameter $\theta$ in (65) is constant; however, for observer design purposes we assume it to be known and in the range

$$
\theta \in[-0.3,0.3]
$$

We choose the premise variables

$$
z_{1, k}=u_{k}, \quad z_{2, k}=\frac{x_{1, k}}{x_{2, k}+b} .
$$

It is evident that $z_{2, k}$ depends on the unmeasured state $x_{2, k}$, making it unmeasured. Assuming a range of values for $u_{k} \in[0,0.4], x_{1, k} \in[0.01,6]$, and $x_{2, k} \in[0.01,3]$, we get the range of the premise variables as

$$
z_{1, k} \in[0,0.4], \quad z_{2, k} \in[0.003,14.63] .
$$




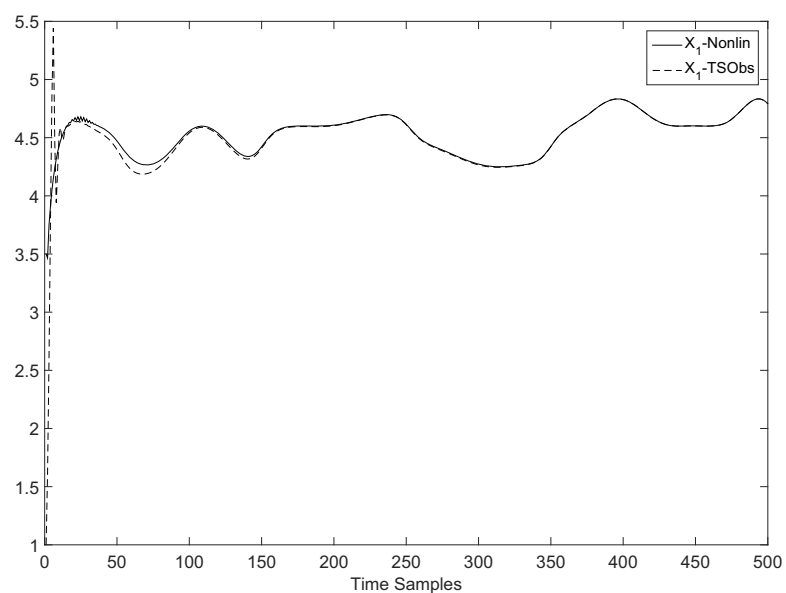

Fig. 1. Estimation of $x_{1, k}$.

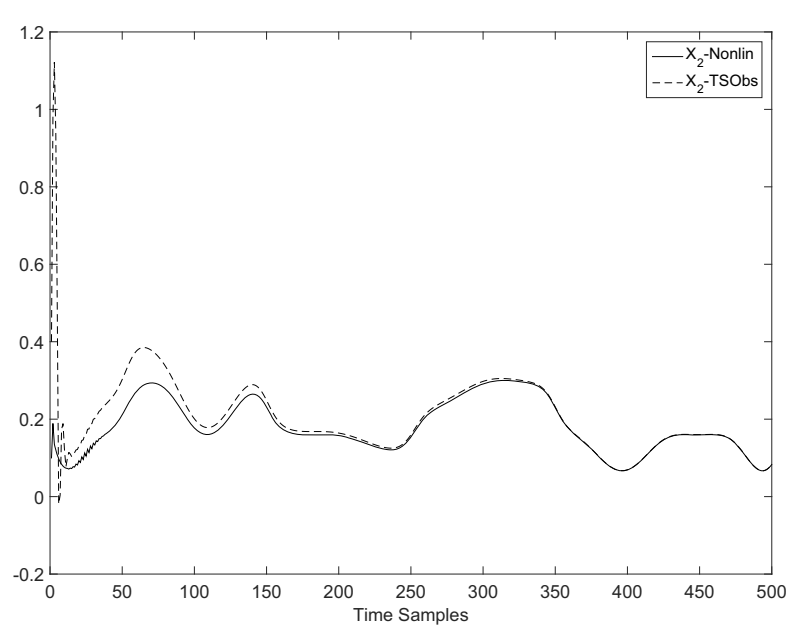

Fig. 2. Estimation of $x_{2, k}$.

With these parameters, we get the model

$$
\mathbf{x}_{k+1}=\sum_{i=1}^{8} h_{i}\left(\mathbf{z}_{k}, \theta_{k}\right)\left[A_{i} \mathbf{x}_{k}+B_{i} u_{k}\right]
$$

where $h_{i}\left(\mathbf{z}_{k}, \theta\right)$ is obtained from the product of membership functions of $z_{1, k}, z_{2, k}$ and $\theta$ corresponding to the submodel $i$. The system matrices are given by

$$
\begin{aligned}
A_{1} & =\left[\begin{array}{cc}
1 & 5.9 \times 10^{-4} \\
0 & 0.99
\end{array}\right], & A_{2} & =\left[\begin{array}{cc}
1 & 0.0024 \\
0 & 0.99
\end{array}\right], \\
A_{3} & =\left[\begin{array}{cc}
1 & 2.92 \\
0 & -0.17
\end{array}\right], & A_{4} & =\left[\begin{array}{cc}
1 & 11.7 \\
0 & -3.68
\end{array}\right], \\
A_{5} & =\left[\begin{array}{cc}
0.6 & 5.9 \times 10^{-4} \\
0 & 0.6
\end{array}\right], & A_{6} & =\left[\begin{array}{cc}
0.6 & 0.0024 \\
0 & 0.6
\end{array}\right],
\end{aligned}
$$

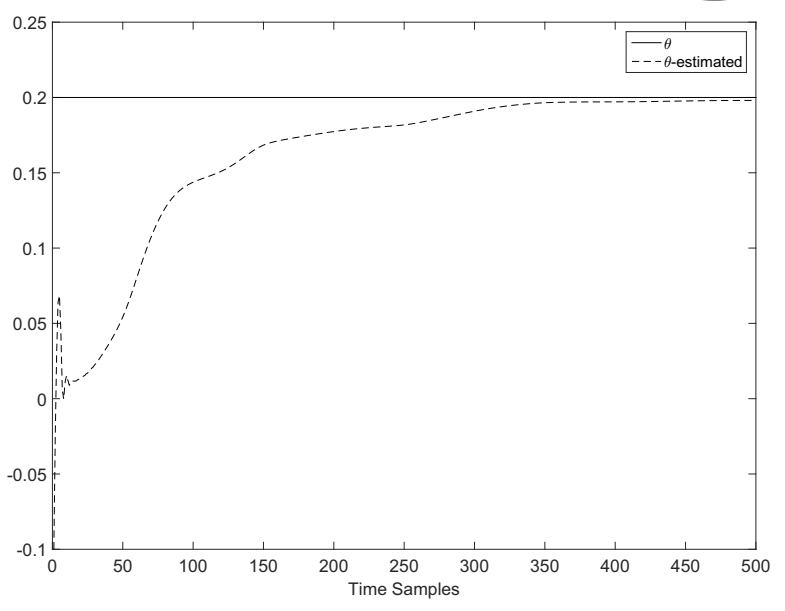

Fig. 3. Estimation of $\theta_{k}$.

$$
\begin{aligned}
& A_{7}=\left[\begin{array}{cc}
0.6 & 2.93 \\
0 & -0.57
\end{array}\right], \quad A_{8}=\left[\begin{array}{cc}
0.6 & 11.7 \\
0 & -4.1
\end{array}\right] \\
& B_{i}=\left[\begin{array}{l}
0 \\
2
\end{array}\right], \quad \forall i \text {. }
\end{aligned}
$$

We used Matlab with the Yalmip (Löfberg, 2004) interface, as well as the LMIlab toolbox, to solve the LMI conditions.

Remark 6. Some problem specific conditions could be added to obtain an optimum solution to the problem. For example, pole placement for the state observers $A_{i}-L_{i} C$ could be added as a separate LMI condition so as to achieve a favourable rate of convergence. Further, some minimum value for the gain corresponding to parameter estimation, $K_{i}, \forall i$ could be imposed so that the innovation term be useful in augmenting the estimated $\theta$ (due to the relative scaling between the values of $\hat{\theta}$ and $y-\hat{y}$ ). Further, as noted in Remark 4 the value of $Q_{e}$ was chosen as

$$
Q_{e}=\left[\begin{array}{cc}
0.001 I_{n_{x}} & 0 \\
0 & 0.1 I_{n_{\theta}}
\end{array}\right] .
$$

Remark 7. It is to be noted that there are a number of variables to be determined by the LMI solver. This could be reduced by fixing some of the parameters. For this example, we chose the values for $\Gamma_{2}^{x}=\Gamma_{2}^{u}=0.1$ and $\lambda_{i}=0.001, \forall i=1,2,3,4$. This significantly reduces the computational complexity of the problem.

With the above conditions, we obtain the following observer gain values:

$$
\begin{aligned}
& L_{1}=\left[\begin{array}{l}
0.23 \\
0.24
\end{array}\right], \quad L_{2}=\left[\begin{array}{l}
0.23 \\
0.24
\end{array}\right], \quad L_{3}=\left[\begin{array}{l}
0.31 \\
0.21
\end{array}\right], \\
& L_{4}=\left[\begin{array}{l}
0.61 \\
0.09
\end{array}\right], \quad L_{5}=\left[\begin{array}{c}
-0.41 \\
0.30
\end{array}\right], \quad L_{6}=\left[\begin{array}{c}
-0.41 \\
0.30
\end{array}\right] \text {, } \\
& L_{7}=\left[\begin{array}{c}
-0.41 \\
0.30
\end{array}\right], \quad L_{8}=\left[\begin{array}{c}
-0.27 \\
0.24
\end{array}\right] \text {, } \\
& \text { and } K_{i}=0.03, \forall i \text {. }
\end{aligned}
$$




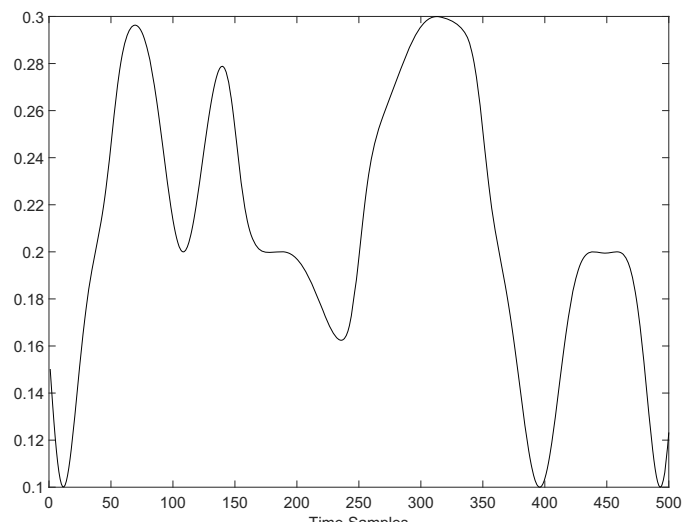

Fig. 4. Input used for the illustrated simulation result.

With these gain values, we obtain the state estimation results as shown in Figs. 1 and 2 (here 'Nonlin' and 'TSObs' refer to the results from nonlinear system T-S observer). The estimation of the unknown parameter is given in (Fig. 3). Further, the input used for the simulation is shown in Fig. 4. To illustrate the nonlinearity of the model, we show the variation of the weighting function $h_{i}(\hat{x}, \hat{\theta})$ in Fig. 5 .

Remark 8. The transient response characteristics of polytopic observers have some known issues. This concerns implicitly taking into account the known bound for the states in observer design. This might make the transient response of estimated states (and hence the parameters) exceed the bounds and lead to jerks in the response as seen in (Fig. 3). This could be partially mitigated through approaches such as that described by Ichalal et al. (2015), but is beyond the scope of this work.

\section{Concluding remarks}

We presented an adaptive observer design procedure for discrete-time nonlinear systems which could be converted to a quasi-LPV form. The work fills a gap in adaptive observer design for discrete-time nonlinear systems for those cases where transformed quasi-LPV matrices depend on one of the unmeasured system states. The results in this approach are conservative, but give way to exploring a systematic approach to adaptive observer design for nonlinear systems of this type. One interesting extension could be to explore other observer structures, especially the one proposed by Guyader and Zhang (2003), and follow a similar design strategy as in our work so as to expand for the unmeasured premise variable case.

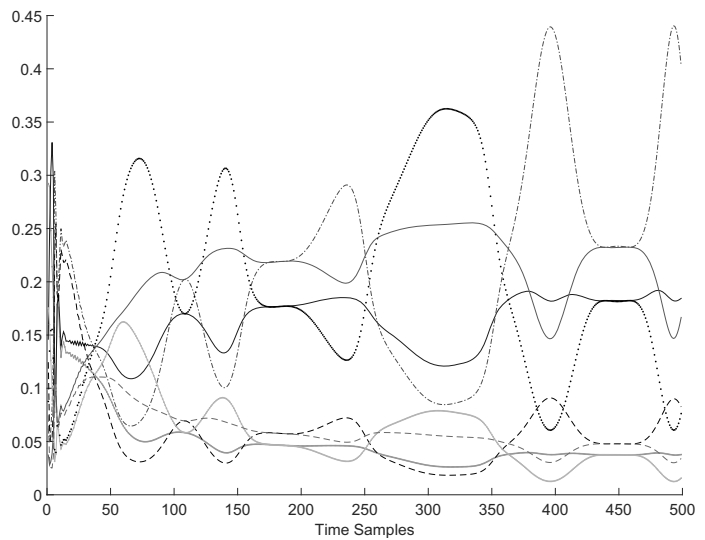

Fig. 5. Weighting functions' evolution for different submodels.

\section{Acknowledgment}

This work was supported by the FP7 project Energy in Time (EiT) under the grant no. 608981.

\section{References}

Bezzaoucha, S., Marx, B., Maquin, D. and Ragot, J. (2013a). State and parameter estimation for nonlinear systems: A Takagi-Sugeno approach, American Control Conference, ACC'2013, Washington, DC, USA, pp. 1050-1055.

Bezzaoucha, S., Marx, B., Maquin, D. and Ragot, J. (2013b). State and parameter estimation for time-varying systems: A Takagi-Sugeno approach, 5th Symposium on System Structure and Control/IFAC Joint Conference 2013 SSSC, Grenoble, France.

Blanco, Y. (2001). Stabilisation des Modeles Takagi-Sugeno et leur usage pour la commande des systemes non lineaires, $\mathrm{PhD}$ thesis, Université des Sciences et Technologies de Lille, Lille.

Boyd, S.P., El Ghaoui, L., Feron, E. and Balakrishnan, V. (1994). Linear Matrix Inequalities in System and Control Theory, SIAM, New York, NY.

Caccavale, F., Pierri, F. and Villani, L. (2008). Adaptive observer for fault diagnosis in nonlinear discrete-time systems, Journal of Dynamic Systems, Measurement, and Control 130(2): 021005.

Cho, Y.M. and Rajamani, R. (1997). A systematic approach to adaptive observer synthesis for nonlinear systems, IEEE Transactions on Automatic Control 42(4): 534-537.

Ţiclea, A. and Besançon, G. (2016). Adaptive observer design for discrete time LTV systems, International Journal of Control 89(12): 2385-2395.

de Souza, C.E. and Xie, L. (1992). On the discrete-time bounded real lemma with application in the characterization of static state feedback $H_{\infty}$ controllers, Systems \& Control Letters 18(1): 61-71.

Guyader, A. and Zhang, Q. (2003). Adaptive observer for discrete time linear time varying systems, 13th 
IFAC/IFORS Symposium on System Identification, SYSID'2003, Rotterdam, The Netherlands, pp. 1705-1710.

Ichalal, D., Mammar, S., Dabladji, M. E.-H. and Ragot, J. (2015). Observer design for a class of discrete-time quasi-LPV systems with unknown parameters: Algebraic approach, 2015 European Control Conference (ECC), Linz, Austria, pp. 915-920.

Ichalal, D., Marx, B., Maquin, D. and Ragot, J. (2016). A method to avoid the unmeasurable premise variables in observer design for discrete time TS systems, International Conference on Fuzzy Systems, FUZZ-IEEE'2016, Vancouver, Canada, pp. 2343-2348.

Ichalal, D., Marx, B., Ragot, J. and Maquin, D. (2009). State and unknown input estimation for nonlinear systems described by Takagi-Sugeno models with unmeasurable premise variables, 17th Mediterranean Conference on Control and Automation, MED'09, Thessaloniki, Greece, pp. 217-222.

Kwiatkowski, A., Boll, M.T. and Werner, H. (2006). Automated generation and assessment of affine LPV models, Proceedings of the 45th IEEE Conference on Decision and Control CDC'06, San Diego, CA, USA, pp. 6690-6695.

Lendek, Z., Guerra, T.M. and De Schutter, B. (2010). Stability analysis and nonlinear observer design using Takagi-Sugeno fuzzy models, Fuzzy Sets and Systems 161(15): 2043-2065.

Löfberg, J. (2004). YALMIP: A toolbox for modeling and optimization in Matlab, International Symposium on Computer Aided Control Systems Design, Taipei, Taiwan, pp. 284-289.

Nagy, A.M., Mourot, G., Marx, B., Ragot, J. and Schutz, G. (2010). Systematic multimodeling methodology applied to an activated sludge reactor model, Industrial \& Engineering Chemistry Research 49(6): 2790-2799.

Ohtake, H., Tanaka, K. and Wang, H.O. (2003). Fuzzy modeling via sector nonlinearity concept, Integrated ComputerAided Engineering 10(4): 333-341.

Srinivasarengan, K., Ragot, J., Maquin, D. and Aubrun, C. (2016a). Joint state and parameter estimation for discrete-time Takagi-Sugeno model, 13th European Workshop on Advanced Control and Diagnosis, ACD 2016, Lille, France, pp. 012016.

Srinivasarengan, K., Ragot, J., Maquin, D. and Aubrun, C. (2016b). Nonlinear joint state-parameter observer for VAV damper position estimation, 3rd Conference on Control and Fault-Tolerant Systems, SysTol 2016, Barcelona, Spain, pp. 164-169.

Tanaka, K. and Wang, H.O. (2004). Fuzzy Control Systems Design and Analysis: A Linear Matrix Inequality Approach, John Wiley \& Sons, New York, NY.

Thumati, B.T. and Sarangapani, J. (2008). A model based fault detection scheme for nonlinear multivariable discrete-time systems, 2008 IEEE International Conference on Systems, Man and Cybernetics, Singapore, pp. 1978-1983.

Zhou, K. and Khargonekar, P.P. (1988). Robust stabilization of linear systems with norm-bounded time-varying uncertainty, Systems \& Control Letters 10(1): 17-20.

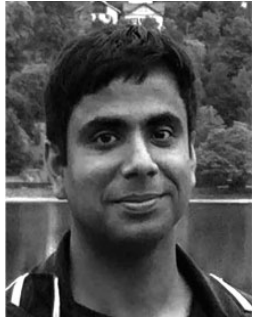

Krishnan Srinivasarengan is a $\mathrm{PhD}$ candidate at Université de Lorraine, Nancy, France, and is associated with the CRAN laboratory (Research Center for Automatic Control in Nancy). He obtained his MTech in control and computing from the Indian Institute of Technology, Bombay, in 2011, and his BTech from the National Institute of Technology, Tiruchirappalli, India. Before starting his doctoral studies, he was a researcher at TCS Innovation Labs, Bangalore, where he is currently on a sabbatical. His broad interests lie in the area of control theory and its applications, with the present focus on observer design.

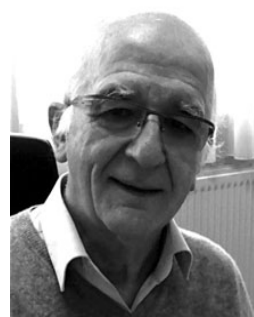

José Ragot received his $\mathrm{PhD}$ in 1973 from the University of Nancy (France). He then joined Henri Poincaré University, Nancy, as an assistant professor, and in 1980 obtained his DSc and in 1985 became a professor at Institut National Polytechnique de Lorraine (INPL), Nancy. He is also a researcher at the Research Centre for $\mathrm{Au}$ tomatic Control in Nancy (CRAN), where he had headed the process diagnosis group for 12 years. Presently, he is a professor emeritus. His interests include data validation and reconciliation, as well as process and fault diagnosis, with a particular focus on multi-models with applications in multiple domains. He has successfully advised $70 \mathrm{PhD}$ candidates and published about 500 refereed technical communications (130 journal articles and 370 conference papers), 4 books, and 14 chapters in collective volumes.

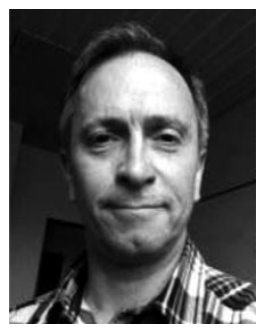

Christophe Aubrun received his $\mathrm{PhD}$ in 1993 from Henri Poincaré University, Nancy I, France. Since 2003 he has been a full professor at Henri Poincaré University, Nancy I, where he teaches automatic control. He has been the director of the University Institute for Training in Electrical Engineering and the head of the Department of Electrical Engineering of the Nancy University Institute of Technology. He is a member of the Research Centre in Automatic Control of Nancy (CRAN). His current research interests are in model-based fault diagnosis and fault tolerant control with the emphasis on networked control systems and continuous commissioning of building energy systems.

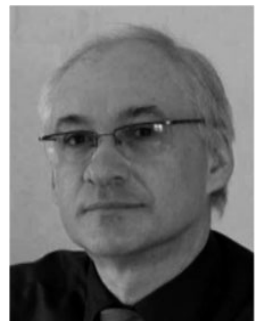

Didier Maquin is a professor of automatic control at the University of Lorraine, France. He teaches automatic control and applied mathematics in various engineering schools. He is a member of the Research Center for Automatic Control of Nancy and the scientific leader of the research department in control, identification and diagnosis, which gathers about forty academics and as many $\mathrm{PhD}$ students. His research topics include state estimation, fault diagnosis and fault tolerant control, both using model-based or data-driven approaches. Didier Maquin has co-authored more than 50 journal articles and 160 conference communications.

Received: 10 February 2017 Revised: 14 June 2017 Accepted: 9 August 2017 